\section{REVISTA}

Actualidades Investigativas en Educación

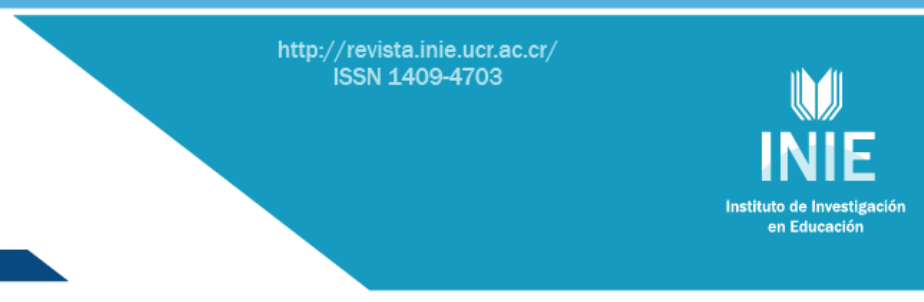

\title{
LA TRANSFERENCIA DE INVESTIGACIÓN EN INSTITUCIONES DE EDUCACIÓN SUPERIOR MEDIANTE SPIN-OFF
}

TRANSFER OF RESEARCH IN HIGHER EDUCATION INSTITUTIONS BY SPIN-OFF

\section{Volumen 15, Número 3}

Setiembre - Diciembre

pp.1-23

\section{Este número se publicó el $1^{\circ}$ de setiembre de 2015}

DOI: http://dx.doi.org/10.15517/aie.v15i3.20983

Mauricio Castillo-Vergara

Alejandro Alvarez-Marin

Revista indizada en REDALYC, $\underline{\text { SCIELO }}$

Revista distribuida en las bases de datos:

CATÁLOGO DE LATINDEX, IRESIE, CLASE, DIALNET, DOAJ, E-REVIST@S, SHERPA/ROMEO, QUALIS, MIAR

Revista registrada en los directorios:

ULRICH'S, REDIE, RINACE, OEI, MAESTROTECA, PREAL, CLACSO 


\title{
LA TRANSFERENCIA DE INVESTIGACIÓN EN INSTITUCIONES DE EDUCACIÓN SUPERIOR MEDIANTE SPIN-OFF \\ TRANSFER OF RESEARCH IN HIGHER EDUCATION INSTITUTIONS BY SPIN-OFF
}

\author{
Mauricio Castillo-Vergara ${ }^{1}$ \\ Alejandro Alvarez-Marin ${ }^{2}$
}

Resumen: Este ensayo tiene como objetivo el análisis de la situación actual respecto al concepto de Spin-Off en Chile como mecanismo que las universidades pueden utilizar para transferir resultados de la investigación pública en el sistema económico. Si bien, se reconoce la importancia en cuanto a la innovación y el emprendimiento, para el desarrollo de universidades emprendedoras, las Spin-Offs no han sido estimuladas desde las universidades. El ensayo se centra en contextualizar el desarrollo de Spin-Off en otros países de Norteamérica y Europa y enfatizar los factores que han permitido su nacimiento, crecimiento y principales resultados en dichas naciones. Su objetivo principal es identificar y proporcionar orientaciones para estimular el desarrollo de Spin-Off universitarias en el país. Se concluye que los modelos de negocio son importantes para comercializar los resultados de investigación, así como el papel que juega el equipo investigador, debiendo considerarse estrategias para fomentar las capacidades propias de este equipo, más allá del perfil técnico. Chile, tiene una gran oportunidad de liderar el desarrollo de esta línea de crecimiento en Latinoamérica, tomando como experiencia los casos internacionales.

Palabras clave: TRANSFERENCIA DE INVESTIGACIÓN, TRANSFERENCIA TECNOLÓGICA, INNOVACIÓN, EMPRENDIMIENTO, EDUCACIÓN SUPERIOR, SPIN-OFF, CHILE.

\begin{abstract}
This essay aims to analyze the current situation regarding the concept of Spin-Off in Chile as a mechanism that universities can use to transfer results of public research into the economic system. Even though the importance of innovation and entrepreneurship is recognized for the development of enterprising universities, the Spin-Off from the universities has not been stimulated. This essay focuses on contextualizing the development of Spin-Off in countries in North America and Europe and emphasize the factors that have allowed its birth, growth and key results in those nations. Its main objective is to identify and provide guidance to stimulate the development of Spin-Off universities in Chile. It is concluded that business models are important for the commercialization of the research results, as is the role that the research team plays, and should consider strategies to encourage the team's own skills in addition to the technical profile. Chile has a great opportunity to lead the development of this line of growth in Latin America, using the experience from the international cases.
\end{abstract}

Keywords: RESEARCH TRANSFER, TECHNOLOGY TRANSFER, INNOVATION, ENTREPRENEURSHIP, HIGHER EDUCATION, SPIN-OFF, CHILE.

\footnotetext{
Académico Departamento de Ingeniería Industrial. Universidad de La Serena, Chile. Magister en Gestión de Empresas. Dirección electrónica: mhcastillo@userena.cl

2 Académico Departamento de Ingeniería Industrial, Universidad de La Serena, Chile. Magister en Tecnologías de la Información. Dirección electrónica: aalvarez@userena.cl
}

Ensayo recibido: 6 de noviembre, 2014

Enviado a corrección: 22 de junio, 2015

Aprobado: 17 de agosto, 2015 


\section{Introducción}

La mayoría de las empresas chilenas no cuentan con los conocimientos, tecnologías y prácticas que utilizan las empresas competitivas de Chile y el mundo en rubros similares (Meneses, 2009). El Instituto Nacional de Propiedad Intelectual (INAPI) afirma que la importancia de la transferencia tecnológica en un país, radica en que esta puede ayudar a la sociedad a solucionar múltiples problemas sociales. En términos de mercado, la transferencia de tecnología puede favorecer la competitividad de las empresas, ya que estas, al adquirir nuevas tecnologías, podrán desarrollar mejores productos y servicios a precios bajos (Instituto Nacional de Propiedad Intelectual, 2012).

De hecho, los países y regiones que acumulan competencias fuertes en innovación y transferencia son más productivos, crecen más rápido y alcanzan mayores niveles de ingreso per cápita (Leten, Landoni y Van Looy, 2014). Los autores Dragan e Isaic-Maniu (2012) afirman que la creación de nuevas empresas y la consolidación del sector privado en la economía es un medio seguro para aumentar el desempeño económico. Es más, las soluciones de mercado como emprendimientos o Spin-Off ofrecen una oportunidad para crear un cambio sustancial y significativamente positivo también dentro de la configuración de la pobreza (Bruton, Ketchen e Ireland, 2013).

Según el informe de Competitividad Global 2014-2015, elaborado por el Foro Económico Mundial (WEF), Chile se ubica en el lugar 34 y las principales conclusiones indicadas señalan que una baja inversión en innovación, especialmente en el sector privado, podría arriesgar la necesaria transición de Chile hacia una economía basada en el conocimiento (Schwab, 2014). Un panel de expertos, a solicitud del Consejo Nacional de Innovación para la Competitividad de Chile, analizó el estado de avance en el país del funcionamiento del Sistema Nacional de Innovación, entre los resultados se destaca que si bien la iniciativa de aumentar el financiamiento de la investigación en la infraestructura del conocimiento en los sectores públicos y privados es ahora más cercano y apropiado a países de la Organización para la Cooperación y el Desarrollo Económicos (OCDE), esta inversión adicional debe centrarse en un cambio de estructura del esfuerzo científico, en lugar de sostener la estructura existente.

El impacto de una mayor inversión en investigación y desarrollo $(I+D)$ en el desarrollo económico se maximiza mediante una mayor armonización de los temas de investigación con objetivos socioeconómicos (Consejo Nacional de Innovación para la Competitividad, 2010). 
Ya desde la década de 1970 se constató la aparición y consolidación de una tercera misión de la universidad: la transferencia de conocimiento al sector industrial, añadiéndose a las misiones tradicionales centradas en la educación y la investigación (Dias y Porto, 2014). El logro de este objetivo implica diversas formas y/o herramientas destinadas al propósito. En la actualidad, la gama de actores institucionales involucrados en actividades de transferencia e innovación es bastante alta. Entre estos actores se encuentran las empresas, la infraestructura tecnológica, incubadoras de empresas, parques científicos, centros tecnológicos, institutos y universidades públicas y privadas de investigación, para los cuales la generación, transferencia y uso del conocimiento y / o tecnología se pueden destacar como actividades clave o insumos esenciales para la innovación (Vedovello y Figueiredo, 2005).

Dentro de esto, en los últimos años se ha visto como alternativa la creación de nuevas empresas a partir de la investigación, como un instrumento de generación de valor a partir de la investigación científica denominado Spin-Off universitario. El objetivo de este ensayo es revisar los resultados de investigaciones que han ampliado el estudio de las Spin-Off. La revisión de estos documentos tiene la finalidad de contextualizar el tema y proporcionar orientaciones que puedan impactar en el diseño de estrategias para que las Universidades en Chile y Latinoamérica incorporen este método de transferencia, dada la importancia que estos mecanismos generan en las economías, en particular sobre su competitividad. Se establece que una cultura dentro de las universidades es relevante para este desarrollo, con ello, se presentan modelos de ecosistemas enfocados a lograr su fortalecimiento al interior de estos centros de educación superior.

\section{Spin-Off}

Las Spin-Off son iniciativas empresariales promovidas por miembros de la comunidad universitaria que se caracterizan por basar su actividad en la explotación de nuevos procesos, productos o servicios a partir del conocimiento adquirido y los resultados obtenidos en la propia Universidad (Beraza y Rodríguez, 2012). La principal diferencia con las Spin-Out es que estas últimas no nacen en el seno de las Universidades, sino de otra organización (Fernández, 2010).

La tabla 1 presenta un cuadro resumen de diversas definiciones del concepto retomadas de un artículo de conceptualización de la Spin-Off universitaria (Beraza y Rodríguez, 2012). 
Tabla 1

Definiciones de Spin-Off

\begin{tabular}{|c|c|}
\hline Autor & Definición Spin-Off Universitaria \\
\hline $\begin{array}{l}\text { Smilor et al. } \\
(1990)\end{array}$ & $\begin{array}{l}\text { "una empresa que es fundada (1) por un profesor, personal de administración y } \\
\text { servicios, o estudiantes que abandonaron la universidad para crear la empresa } \\
\text { o que comenzaron la empresa mientras todavía eran miembros de la } \\
\text { universidad; y/o (2) en torno a una tecnología o una idea basada en una } \\
\text { tecnología desarrollada dentro de la universidad". }\end{array}$ \\
\hline $\begin{array}{l}\text { Giannisis et al. } \\
\text { (1991) }\end{array}$ & $\begin{array}{l}\text { "una empresa que produce un producto o un servicio derivado de la } \\
\text { investigación que se realiza en una universidad". }\end{array}$ \\
\hline Doutriaux (1992) & $\begin{array}{l}\text { "una Spin-Off debe haber sido creada por un universitario (profesor, } \\
\text { investigador, estudiante), sólo o con la ayuda de la universidad, con vistas a la } \\
\text { explotación comercial de una idea o de una invención universitaria". }\end{array}$ \\
\hline $\begin{array}{l}\text { Carayannis et } \\
\text { al. (1998) }\end{array}$ & $\begin{array}{l}\text { "una nueva empresa creada por individuos que son antiguos empleados de una } \\
\text { organización madre (la universidad), en torno a una tecnología que se originó } \\
\text { en la organización madre y que fue transferida a la nueva empresa". }\end{array}$ \\
\hline $\begin{array}{l}\text { Bellini et al. } \\
(1999)\end{array}$ & $\begin{array}{l}\text { "Spin-Offs académicas son empresas fundadas por profesores, investigadores, } \\
\text { o estudiantes y graduados universitarios para explotar comercialmente los } \\
\text { resultados de la investigación en la que han estado implicados en la } \\
\text { universidad". }\end{array}$ \\
\hline $\begin{array}{l}\text { O'Gorman y } \\
\text { Jones-Evans } \\
\text { (1999) }\end{array}$ & $\begin{array}{l}\text { "la formación de una nueva empresa u organización para explotar los } \\
\text { resultados de la investigación universitaria". }\end{array}$ \\
\hline $\begin{array}{l}\text { Klofsten } \quad y \\
\text { Jones-Evans } \\
\text { (2000) }\end{array}$ & $\begin{array}{l}\text { "formación de una nueva empresa para explotar los resultados de la } \\
\text { investigación universitaria". }\end{array}$ \\
\hline $\begin{array}{l}\text { Braun et al. } \\
(2000)\end{array}$ & $\begin{array}{l}\text { "las Spin-Offs son definidas como nuevas empresas formadas por un individuo } \\
\text { o un grupo de individuos que son antiguos o actuales empleados de una } \\
\text { universidad y que establecen la empresa en torno a una tecnología o una idea } \\
\text { de negocio con origen en la investigación universitaria". }\end{array}$ \\
\hline $\begin{array}{l}\text { Clarysse Tartari } \\
\text { y Ammon (2011) }\end{array}$ & $\begin{array}{l}\text { "Spin-Offs basadas en la investigación son nuevas empresas establecidas por } \\
\text { una institución anfitriona (universidad, escuela técnica, departamento de I+D } \\
\text { público/privado) para transferir y comercializar invenciones resultantes de los } \\
\text { esfuerzos de I+D de los departamentos". }\end{array}$ \\
\hline Thorburn (2001) & $\begin{array}{l}\text { "una Spin-Off es una empresa establecida para comercializar tecnología de una } \\
\text { universidad, la cual licencia tecnología de la institución, y a la que pasa } \\
\text { personal de la institución de una forma temporal o permanente". }\end{array}$ \\
\hline Degroof (2002) & $\begin{array}{l}\text { "las características más importantes que permiten definir a una Spin-Off } \\
\text { académica son la transferencia de tecnología de una institución de } \\
\text { investigación y el hecho de que la empresa es nueva, establecida con el fin de } \\
\text { explotar esta tecnología". }\end{array}$ \\
\hline Cotec (2003) & $\begin{array}{l}\text { "las iniciativas empresariales en las que el fundador/a pertenece a alguno de } \\
\text { los estamentos de la universidad (profesores, personal técnico o administrativo } \\
\text { e investigadores), o bien se crea en base al conocimiento o tecnología creada y } \\
\text { propiedad de la institución". }\end{array}$ \\
\hline Golob (2003) & $\begin{array}{l}\text { "Spin-Off se refiere a nuevas empresas formadas en torno a una tecnología por } \\
\text { individuos que son antiguos empleados de la organización madre donde se } \\
\text { originó la tecnología". }\end{array}$ \\
\hline
\end{tabular}




\begin{tabular}{|l|l|}
\hline $\begin{array}{l}\text { Tang et al. } \\
(2004)\end{array}$ & $\begin{array}{l}\text { "una Spin-Off universitaria es una nueva empresa cuya formación depende de } \\
\text { los derechos de la propiedad intelectual de la universidad y en la que la } \\
\text { universidad tiene una participación en el capital". }\end{array}$ \\
\hline $\begin{array}{l}\text { McDonald et al. } \\
(2004)\end{array}$ & $\begin{array}{l}\text { "el término Spin-Off universitaria es definida como una nueva empresa creada } \\
\text { principalmente con el propósito de explotar tecnología desarrollada } \\
\text { originalmente por la universidad. En la mayoría de los casos, personal científico } \\
\text { implicado en la creación de esta tecnología deja la universidad y se hace } \\
\text { miembro de la nueva empresa, logrando de este modo la transferencia de } \\
\text { tecnología. Esta transferencia de personal puede realizarse de forma temporal } \\
\text { o permanente. Además, muchos de los inventores académicos principales } \\
\text { juegan algún papel en la nueva empresa pero mantienen su puesto } \\
\text { académico". }\end{array}$ \\
\hline $\begin{array}{l}\text { Lockett y Wright } \\
\text { (2005) }\end{array}$ & $\begin{array}{l}\text { "nosotros definimos de forma restringida las Spin-Out universitarias como } \\
\text { nuevas empresas que dependen de la licencia o asignación de la propiedad } \\
\text { intelectual de la institución para su iniciación". }\end{array}$ \\
\hline $\begin{array}{l}\text { Grandi } \\
\text { Grimaldi (2005) }\end{array}$ & $\begin{array}{l}\text { "una definición genérica de Spin-Off abarca los casos en los que académicos } \\
\text { crean una empresa con base en una tecnología propiedad de la universidad } \\
\text { (licencia de una tecnología patentada) o a un conocimiento tecnológico más } \\
\text { genérico (no propiedad de la universidad). También incluye situaciones en las } \\
\text { que la universidad opta por conceder los derechos de explotación de la } \\
\text { tecnología a un externo, independiente emprendedor, no perteneciente a la } \\
\text { universidad (fundador no académico), quien pone en marcha una nueva } \\
\text { empresa". }\end{array}$ \\
\hline
\end{tabular}

Fuente: Beraza y Rodríguez (2012)

\subsection{Spin-Off y Universidades}

La puesta en marcha de proyectos empresariales (Spin-Off) es un mecanismo que las universidades pueden utilizar para transferir resultados de la investigación pública en el sistema económico. Es un canal importante por varias razones: Di Gregorio y Shane (2003) sostienen que existe una relación positiva entre la generación de Spin-Off y el prestigio de las universidades. Rasmussen y Wright (2015) destacan que son dos los argumentos de porque las universidades deben priorizar en la creación de ellas; el primero, se relaciona con el impacto económico directo, pues ayudan a generar nuevos empleos, ingresos fiscales y posibilidad de competir internacionalmente; el segundo, consiste en pueden actuar como mecanismo de transferencia de tecnología y conocimiento, y es visto como una herramienta para facilitar la difusión.

Por su parte, Walter, Auer y Ritter (2006) señalan las siguientes razones: Spin-Offs universitarios pueden verse desde diferentes perspectivas como fuentes de empleo, como mediadores de investigación básica e investigación aplicada que permitan a sus clientes competir en la vanguardia tecnológica, como contribuyentes a una mayor eficiencia de la innovación, como un aporte al desarrollo económico de una región o como agentes de cambio del panorama económico. 
El desarrollo de estos tipos de transferencia no solo proporciona los beneficios indicados, los autores Soetanto y Van Geenhuizen (2015) han demostrado que existe una relación positiva con la capacidad que tienen las Spin-Off para el atraer financiamiento a las Universidades. Un estudio realizado por el Banco de Boston en 1997 identificó 4000 empresas Spin-Off del Massachusetts Institute of Technology (MIT) que emplearon 1,1 millones de personas y generaron $\$ 232.000$ millones en ventas anuales en todo el mundo (Steffensen, Rogers y Speakman, 2000).

Sin embargo, varias investigaciones empíricas muestran que la mayoría de los sectores derivados de la investigación, no logran desarrollarlo de forma exitosa en países fuera de Europa o América del Norte (Visintin y Pittino, 2014). Ante esto, dos modelos de negocio pueden ser utilizados: el primero, optar por vender o licenciar su tecnología; el segundo, decidir seguir con el desarrollo de productos o servicios basados en la investigación, lo cual es una importante opción estratégica para la creación de empresas Spin-Off (Conceição, Fontes y Calapez, 2012).

Conscientes los gobiernos de un consenso general en el valor de promover la comercialización del conocimiento y la investigación generada en las universidades para impactar en el desarrollo de las economías, promueven políticas en dicho sentido. Se han generado cambios en la legislación que regula la transferencia de tecnología universitaria, tales como la promulgación de la ley Bayh-Dole en los Estados Unidos de Norteamérica (EEUU), lo que significó que casi todas las principales universidades de investigación de ese país, establecieran una oficina de transferencia de tecnología, con una mayor atención a las patentes académicas y para la concesión de licencias de los resultados. Fuera de los EE.UU., los cambios en la comercialización universitaria están vinculados a reformas más generales.

Ejemplos de ello son la reforma de todo el sistema académico introducido en el Reino Unido y en los Países Bajos durante los años 1990, la llamada Loi Allègre de 1999 en Francia, también, el Gobierno de Suecia realiza esfuerzos para promover la comercialización de tecnología universitaria desde el comienzo de la década de 1980. En Italia se genera el traslado de varios poderes del Gobierno central a las universidades, en Dinamarca, Alemania, Austria y Noruega reformaron sus leyes de propiedad intelectual para conceder derechos de propiedad intelectual a las universidades, esto, de una manera similar a la Ley Bayh-Dole. 
Otras naciones están considerando reformas similares (Grimaldi, Kenney, Siegel y Wright, 2011). En Latinoamérica, aún no existe una regulación sobre la creación de empresas Spin-Off, lo que puede ocasionar conflictos de interés, entre académicos e investigadores, aun cuando ellos no sean dueños de dichas empresas (Dias y Porto, 2014), y tanto los autores Goldfarb y Henrekson (2003) como Bacchiocchi y Montobbio (2009) establecen que la legislación vigente de los países puede constituirse en un desincentivo a la creación de este tipo de empresas.

Uno de los casos más exitosos y más antiguos de creación y desarrollo de Spin-Off académicas es el de EEUU, país en el cual la legislación promueve un ambiente propicio para el emprendimiento universitario y cuya evolución ha permitido que las universidades líderes desarrollen estrategias para vincularse con el entorno, constituyéndose en centros interactivos para las empresas y demás organizaciones de la sociedad que buscan soluciones, por ello, se consideran pioneros en innovación colaborativa. En el periodo 19802005 se reportó la creación de más de 4.543 empresas biotecnológicas en las universidades, hospitales y centros de investigación estadounidenses, la introducción en el mercado de unos 50 productos de alta tecnología y un aumento exponencial de patentes concedidas con tendencia media de $8 \%$ superior con respecto al año precedente, según la revista anual de la Association of University Technology Managers (Jiménez, Maculan, Otálora, Moreira y Castellanos, 2013).

En cuanto al proceso global de valorización de las Spin-Off al interior de las universidades, Ndonzuau, Pirnay y Surlemont, (2002) identificaron cuatro etapas relevantes para explicar la transformación de los resultados de la investigación académica sobre el valor económico. En la figura 1 se muestra el modelo de insumo-producto con las respectivas cuatro etapas.

Figura 1

El proceso global de valorización por Spin-Off

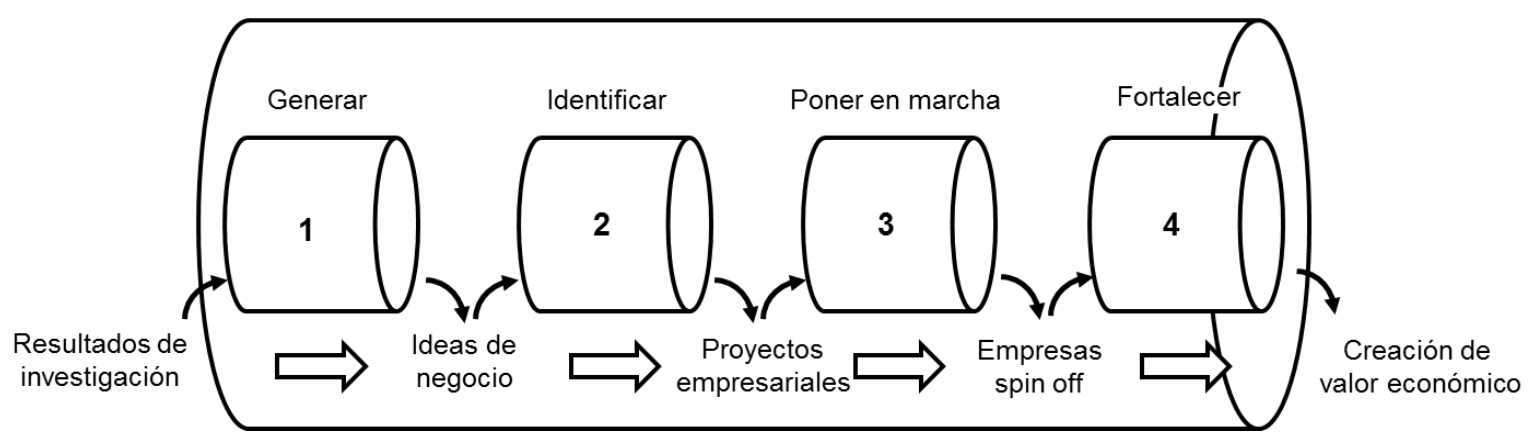

Fuente: Ndonzuau, Pirnay y Surlemont (2002) 
Las etapas identificadas por los autores, son las siguientes:

Etapa 1: Esta etapa se caracteriza por generar ideas de negocio a partir de la investigación. El objetivo de la primera etapa, es producir ideas de negocio, sugerencias y propuestas dentro de la comunidad científica para la explotación comercial. Para la mayoría de las universidades, el realizar negocios con su investigación, requiere cambios radicales en la forma en que han explotado tradicionalmente sus resultados. En efecto, este tipo de explotación comercial implica, necesariamente, aproximar dos conceptos tradicionalmente opuestos en la ciencia: la concepción "científica" que considera la ciencia como un fin en sí mismo, y la concepción "económica" que la considera como un medio para lograr otros objetivos (en particular, la obtención de recursos). Por consiguiente, uno de los principales problemas en este aspecto es clarificar cómo conciliar estas dos concepciones opuestas.

Etapa 2: Esta etapa se singulariza por identificar nuevos proyectos empresariales. Las ideas generadas en la primera etapa del proceso, generalmente, no presentan un foco muy claro, por lo que estas deben ser aclaradas. Tales ideas consisten en elementos técnicos y científicos, mientras que su potencial de negocio no se conoce con precisión. Ese potencial debe ser validado en un proyecto empresarial. Por tanto, el objetivo de la segunda fase es la de transformar una idea incipiente en un proyecto coherente y estructurado de creación de empresas, identificando dos interrogantes específicas: la protección y el desarrollo de la idea. Este paso puede requerir de grandes inversiones, y significa el primer hito importante de la investigación enfocada al ámbito empresarial.

Etapa 3: En esta fase se pretende poner en marcha las empresas Spin-Off de los proyectos. Al finalizar la segunda etapa del proceso, el nuevo proyecto de empresa debe estar listo. La tercera etapa se enfoca en que esta nueva empresa explote la oportunidad de negocio gestionado por un equipo de profesionales y el apoyo de los recursos disponibles. Estos son los tres pilares fundamentales de cualquier éxito empresarial. Los temas tratados serán progresivamente alejados de contingencias académicas específicas hacia consideraciones comerciales. Es importante señalar dos problemas en esta etapa: la disponibilidad de recursos y las relaciones que deben establecerse entre la empresa Spin-Off universitaria y su madre. Con el fin de superar estos problemas, algunas universidades están activamente interesadas en la búsqueda de soluciones, entre las que se encuentra la recaudación de fondos de capital de riesgo, aunque otras instituciones consideran estos problemas ajenos a su misión y ámbito de competencia. 
Etapa 4: En esta etapa se busca fortalecer la creación de valor económico de las empresas Spin-Off. Si bien, la creación de nuevas empresas de base tecnológica es un paso fundamental en la valorización de los Spin-Off universitarios no es el último paso. Se debe tener una amplia perspectiva de la finalidad última del proceso además de la creación de valor económico. Por lo anterior, se debe propiciar la generación de ventajas tangibles e intangibles para la economía local.

En tanto, Beraza y Rodríguez (2011), afirman que la implicación de la universidad resulta indispensable en las primeras etapas del proceso de creación de Spin-Off, y que gradualmente va perdiendo importancia, lo que no quiere decir que la universidad no se puede implicar en las últimas etapas, sino que existen otros agentes (entidades financieras, parques científicos y tecnológicos, incubadoras de empresas, etc.) que pueden favorecer el desarrollo de las mismas de forma más eficaz y eficiente (figura 2).

Figura 2

Proceso de creación de Spin-Off: etapas del proceso e implicación de la Universidad

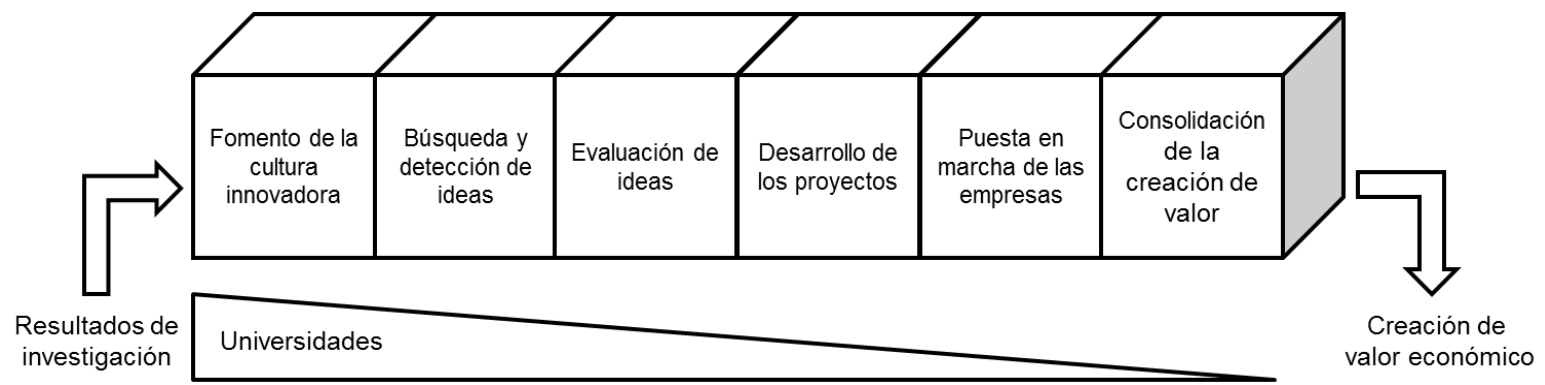

Fuente: Beraza y Rodríguez (2011).

A su vez Iglesias, Jambrino y Peñafiel (2012), identifican tres conglomerados al caracterizar las Spin-Off universitarias, los cuales se describen a continuación:

El primer conglomerado se caracteriza por representar a las Spin-Off universitarias que soportan parte de sus gastos en I+D e innovación, utilizando la financiación pública como recurso de apoyo. Este grupo de empresas muestra unos índices superiores en los indicadores relativos a la inversión en I+D e innovación y en la capacidad de generar resultados de investigación, objeto de gestión de la propiedad industrial. La dimensión de las Spin-Off universitarias, incluidas en este primer conglomerado, alcanza la categoría de pequeña y mediana empresa en cuanto a personal empleado en la organización. Destaca en este grupo la existencia de un valor inferior en cuanto a disposición a participar en redes, esto, en comparación con los otros conglomerados. Por su parte, el número de ingresos es 
proporcional al valor de gastos en I+D e innovación. Finalmente, la productividad en proyectos en este grupo es alta pero no superior en términos absolutos, en comparación con los otros dos conglomerados.

El segundo conglomerado representa a un conjunto de Spin-Offs universitarias que utilizan en menor proporción la financiación pública como recurso de apoyo. Éstas muestran una correspondencia en cuanto al menor nivel en términos de propiedad industrial generada, índice de productividad en número de proyectos y sumatorio de ingresos derivados de la explotación directa de los resultados de la investigación (ingresos por patentes y modelos de utilidad, ingresos por licencias de tecnología y asesoramiento científico-técnico a terceros). Además, en el segundo conglomerado se concentran empresas con un número de trabajadores menor, lo que permite clasificarlas como pequeñas o medianas. La disposición para el establecimiento de relaciones y participación en redes es mayor que para el conglomerado anterior.

El tercer conglomerado recoge a las Spin-Off universitarias menos consolidadas en términos de desarrollo de la actividad de I+D e innovación. Estas Spin-Off no reciben financiamiento público y presentan valores inferiores respecto al primer y segundo conglomerado en gastos en I+D e innovación, sumatorio de variables relativas a propiedad industrial (patentes, modelos de utilidad y tecnología licenciada) e ingresos percibidos de la actividad de I+D. Asimismo, la productividad medida en número de proyectos ejecutados muestra una capacidad limitada. Este índice de número de proyectos menor es coherente puesto que las empresas agrupadas en el tercer conglomerado tienen una proporción inferior tanto en número de trabajadores como en volumen de facturación. En todo caso, conviene subrayar que el establecimiento de relaciones y la participación en redes con agentes del Sistema Nacional de I+D es más alto que para otros conglomerados.

El cuadro resumen de caracterización de los tres conglomerados se muestra en la tabla 2. 
Tabla 2

Caracterización de las Spin-Off

\begin{tabular}{|c|c|c|c|}
\hline Tipo Spin-Off & $\begin{array}{l}\text { Estructura de la } \\
\text { organización }\end{array}$ & Recursos & Apoyos \\
\hline $\begin{array}{l}\text { Spin-Off } \\
\text { Consolidada }\end{array}$ & \begin{tabular}{lrr} 
Suficiente & \multicolumn{2}{r}{ equipo } \\
humano propio & y \\
apoyo externo & al \\
desarrollo & & de \\
proyectos & &
\end{tabular} & $\begin{array}{l}\text { Capacidad de } \\
\text { autofinanciación } \\
\text { Recursos financieros } \\
\text { propios en aumento } \\
\text { Incentivos y/o } \\
\text { participación externa } \\
\text { en capital importante }\end{array}$ & $\begin{array}{lr}\text { Red } & \text { de } \\
\text { colaboraciones } & \text { y } \\
\text { relación con agentes } \\
\text { del sistema de } 1+D+i \\
\text { fluida pero no } \\
\text { dependiente }\end{array}$ \\
\hline $\begin{array}{l}\text { Spin-Off en } \\
\text { Desarrollo/ } \\
\text { Crecimiento }\end{array}$ & $\begin{array}{l}\text { En aumento } \\
\text { Inicio de colaboración } \\
\text { con otros equipos } \\
\text { para desarrollo de } \\
\text { proyectos }\end{array}$ & $\begin{array}{l}\text { Superando umbral de } \\
\text { rentabilidad pero } \\
\text { relativa dependencia } \\
\text { de incentivos, capital } \\
\text { de riesgo y apoyos } \\
\text { para desarrollo de } \\
\text { actividad de I+D+i }\end{array}$ & $\begin{array}{l}\text { Red de } \\
\text { colaboraciones y } \\
\text { relación con agentes } \\
\text { del sistema de l+D+i } \\
\text { Protagonismo en red } \\
\text { de la universidad }\end{array}$ \\
\hline $\begin{array}{l}\text { Spin-Off } \\
\text { Start-Up }\end{array}$ & $\begin{array}{lr}\text { Reducida } & \text { tanto en } \\
\text { cuanto a personal } \\
\text { como } & \text { en } \\
\text { equipamiento. etc. } & \end{array}$ & $\begin{array}{lr}\text { Endeudamiento } & \text { o } \\
\text { dependencia } & \text { de } \\
\text { apoyo externo para el } \\
\text { desarrollo de la } \\
\text { actividad } \\
\text { Los proyectos de } \\
\text { l+D+i se pueden ver } \\
\text { supeditados } & \text { a } \\
\text { existencia } & \text { de } \\
\text { financiación externa } \\
\text { de apoyo }\end{array}$ & $\begin{array}{l}\text { Red de } \\
\text { colaboraciones y } \\
\text { relación con agentes } \\
\text { del sistema de I+D+i } \\
\text { Protagonismo en red } \\
\text { de la universidad y } \\
\text { parques científicos }\end{array}$ \\
\hline
\end{tabular}

Fuente: Iglesias et al. (2012)

Algunas proposiciones relacionadas con Spin-Off, que podrían ser objeto de contraste en estudios empíricos, según Beraza y Rodríguez (2010, p. 132), son:

1. El espíritu emprendedor, la experiencia profesional previa y la implicación del inventor favorecen la transferencia de conocimiento mediante la creación de Spin-Off.

2. La concesión de licencias exclusivas, la toma de participaciones en el capital de las Spin-Off, la flexibilidad de la política de personal, el acceso a los recursos universitarios, una adecuada distribución de los royalties y la oferta de capital semilla por parte de la universidad favorecen la transferencia de conocimiento mediante la creación de Spin-Off.

3. Las tecnologías radicales, que incorporan conocimiento tácito, que se encuentran en sus etapas iniciales, que son de propósito general, que tienen un significativo valor para el cliente, que suponen un importante avance técnico y que tienen una fuerte 
protección de la propiedad intelectual, es más probable que sean comercializadas por medio de Spin-Off.

4. Los resultados de investigación directamente comercializables, con un horizonte temporal de comercialización largo y que requieren inversiones relativamente reducidas, son más probable que sean comercializados por medio de Spin-Off.

5. Los resultados de investigación comercializables en sectores con fuerte protección de la propiedad intelectual, con poca necesidad de activos complementarios, con una base tecnológica joven, con mercados segmentados y con un tamaño reducido de las empresas, son más probable que sean comercializados por medio de Spin-Off.

6. La creación de Spin-Off es más rentable que la concesión de licencias a empresas establecidas para la universidad.

7. Una universidad con una persona de prestigio y experiencia en la industria a la cabeza de la unidad de transferencia tecnológica, que tiene una larga experiencia en dicha actividad, que realiza la misma a través de unidades privadas con ánimo de lucro y que tiene un personal en la unidad con conocimientos y habilidades en las áreas tecnológica y de marketing, favorece la utilización de este mecanismo de transferencia.

\subsection{Focos de estudio de los Spin-Off}

A partir de la revisión bibliográfica se identificaron cinco focos de análisis relacionados con los Spin-Off, enunciados a continuación y cuyos autores se presentan en la tabla 3 :

1. Ecosistema Asociado a los Spin-Off. Un ecosistema tiene sus raíces en la idea de las redes de valor y puede ser visto como un grupo de actores, entre ellos Empresas, Universidades, Estado, Comunidad, Emprendedores, Académicos, etc., que crean valor de forma simultánea mediante la combinación de sus habilidades y activos (Clarysse, Wright, Bruneel y Mahajan, 2014).

2. Estrategia de Comercialización de Spin-Off. El término se utiliza en torno a la propuesta de valor de la empresa, es decir, los segmentos del mercado, los objetivos de la empresa y el valor que crea para sus clientes (Tongur y Engwall, 2014).

3. Factores de Éxito de las Spin-Off. El éxito puede definirse como el incremento de ventajas competitivas para las empresas a través de la generación de valor de sus actividades (García y Mulero, 2007).

4. Otros Mecanismos de Transferencia Tecnológica. La transferencia tecnológica implica diversas formas y/o herramientas destinadas al propósito, diferentes de los Spin-Off en 
estudio. Algunos ejemplos de ellos son: Consultoría, cooperación en el desarrollo de productos, otorgamiento de patentes o licencias, subcontrato para desarrollo de productos, capacitación, oficinas de transferencia tecnológica, parques científicos, incubadora de negocio.

5. Regulación, Leyes y Políticas para el desarrollo de Spin-Off. Diversas economías mundiales han generado cambios en la legislación, regulación, leyes y/o políticas reguladoras de la transferencia de tecnología universitaria.

\section{Tabla 3}

Focos de Análisis de los Manuscritos Revisados

\begin{tabular}{|c|c|}
\hline Focos & Autores \\
\hline Ecosistema Asociado a los Spin-Off & $\begin{array}{l}\text { (Agarwal y Shah Sonali, 2014); (Aldridge y Audretsch, } \\
\text { 2011); (Casper, 2013); (Clarysse et al., 2014); } \\
\text { (Clarysse et al., 2011); (Colombo y Piva, 2012); } \\
\text { (Crespi, D'Este, Fontana y Geuna, 2011); (Kenney y } \\
\text { Patton, 2011); (Leten et al., 2014); (Sternberg, 2014); } \\
\text { (Treibich, Konrad y Truffer, 2013); (Van Looy, Landoni, } \\
\text { Callaert, Van Pottelsberghe, Sapsalis y Debackere, } \\
\text { 2011); (Wennberg, Wiklund, Johan y Wright, 2011) }\end{array}$ \\
\hline $\begin{array}{l}\text { Estrategia de Comercialización de } \\
\text { Spin-Off }\end{array}$ & $\begin{array}{l}\text { (Astebro, Bazzazian y Braguinsky, 2012); (Buenstorf y } \\
\text { Schacht, 2013); (Conceição et al., 2012); (Genet, } \\
\text { Errabi y Gauthier, 2012); (Lehoux, Daudelin, Williams- } \\
\text { Jones, Denis y Longo, 2014); (O’Kane, Mangematin, } \\
\text { Geoghegan y Fitzgerald, 2014) }\end{array}$ \\
\hline Factores de Éxito de las Spin-Off & $\begin{array}{l}\text { (Acosta, Coronado y Martínez., 2012); (Gilsing, } \\
\text { Bekkers, Bodas y Van der Steen, 2011); (Grimaldi et } \\
\text { al., 2011); (Rasmussen, Mosey y Wright, 2014); } \\
\text { (Visintin y Pittino, 2014) }\end{array}$ \\
\hline $\begin{array}{l}\text { Otros Mecanismos de Transferencia } \\
\text { Tecnológica }\end{array}$ & $\begin{array}{l}\text { (Bozeman, Rimes y Youtie, 2014); (Ortín-Angel y } \\
\text { Vendrell-Herrero, 2014); (Vedovello y Figueiredo, } \\
\text { 2005) }\end{array}$ \\
\hline $\begin{array}{l}\text { Regulación, Leyes y Políticas para el } \\
\text { desarrollo de Spin-Off }\end{array}$ & $\begin{array}{l}\text { (Dias y Porto, 2014); (Fini, Grimaldi, Santoni y Sobrero, } \\
2011 \text { ) }\end{array}$ \\
\hline
\end{tabular}

Fuente: Elaboración propia.

\section{Discusión}

En el estudio de Ortín-Ángel y Vendrell-Herrero (2014), se ha demostrado que las capacidades sustantivas de Spin-Off universitarias en el primer año de operación son significativamente inferiores a las de otros mecanismos de transferencia tecnológica, sin embargo, en el quinto año de vida las Spin-Off universitarias presentan mejores resultados. En resumen, los resultados sugieren que en el largo plazo, los Spin-Off universitarios tienen una mayor capacidad para el desarrollo de modelos de negocio de creación de riqueza que 
en el caso de otros mecanismos de transferencia. Es relevante, entonces, que las universidades generen políticas y mecanismos adhoc a la creación de Spin-Off, así también como reglamentos de colaboración externa y regulación de patentes (Fini, Grimaldi, Santoni y Sobrero, 2011).

Es relevante considerar la importancia del diseño de modelo de negocios centrado en el usuario y cómo desde la investigación se debe aprender de estos procesos (Lehoux, Daudelin, Williams-Jones, Denis y Longo, 2014), en lugar de únicamente utilizar la reputación científica de la Universidad. La construcción de relaciones y el respeto innato por la ciencia académica como medio de combinar la ciencia y los negocios de parte de los ejecutivos de los Spin-Off pueden mejorar los resultados (O’Kane, Mangematin, Geoghegan y Fitzgerald, 2014). Se debe considerar en el diseño del modelo, el área científica a desarrollar en el SpinOff, pues si bien en EEUU, la Ley Bayh-Dole en 1980 trajo cambios legislativos y organizativos importantes en el proceso de creación de Spin-Off, los cuales permitieron a las universidades estadounidenses aumentar su número, estas tuvieron bastante éxito en el contexto de la biotecnología pero las políticas para ciencias de nanotecnologías debieron ser diseñadas de manera diferente (Genet, Errabi y Gauthier, 2012). El campo de ciencias de la computación e ingeniería eléctrica genera un mayor número de Spin-Off que otras dos categorías: las ciencias biomédicas y el campo de la ingeniería (Kenney y Patton, 2011).

Como factores de éxito, Visintin y Pittino (2014) señalan la importancia de una composición equilibrada en cuanto a los miembros académicos y no académicos, pues tienen también implicaciones relevantes para la gestión. Así, tiene un impacto importante en el desarrollo sostenible de los proyectos, el apoyo inicial de los departamentos y sus autoridades, en la adquisición de la experiencia comercial necesaria. Emprendimientos apoyados por universidades ayudaron a desarrollar competencias empresariales e influyeron en cómo se desarrollaron estas competencias de actores externos fuera de ella.

También se puede constatar que la falta de apoyo para la iniciativa empresarial limitó gravemente la evolución de las Spin-Off, independientemente de las políticas de la Universidad (Rasmussen, Mosey y Wright, 2014). De la misma forma los autores Grimaldi et al. (2011) señalan que son necesarios para su desarrollo, tres niveles: (a) las especificidades a nivel de sistema (acciones gubernamentales, configuraciones institucionales, las características locales de contexto, etc.); (b) mecanismos de apoyo interno de nivel universitario; y (c) los factores individuales del equipo emprendedor. 
El contexto regional en el que un individuo comienza una Spin-Off universitaria tiene un impacto en el éxito de puesta en marcha, pero el hecho de que él o ella haya recibido el apoyo del gobierno tiene un impacto menor (Sternberg, 2014). Las universidades con una productividad científica más fuerte parecen encontrarse en una posición ventajosa para el desarrollo de actividades empresariales, pues los hallazgos sugieren que los socios industriales tienen en cuenta la producción científica de las universidades como criterio para la selección de socios académicos (Van Looy et al., 2011) y los contactos directos entre científicos universitarios y científicos del sector son un motor importante de los procesos de comercialización académicas (Casper, 2013), pues se generan efectos positivos tanto de las actividades de investigación educativa y científica de las universidades sobre el desempeño tecnológico de las empresas adyacentes (Leten et al., 2014).

Un diverso ecosistema de innovación puede ser necesario para la creación y desarrollo de la industria porque los empleados, académicos y usuarios fundan empresas utilizando conocimiento distinto y necesario para la industria. Además, la función de cada contexto del conocimiento como fuente de la innovación empresarial evoluciona a medida que las industrias maduran (Agarwal y Shah Sonali, 2014). Sin embargo, resultan relevantes los atributos a nivel individual y la experiencia como predictores de éxito más importantes en la iniciativa empresarial académica, aunque el entorno social que rodea al académico también juega un papel importante, pero su papel es mucho menos pronunciada que los factores a nivel individual (Clarysse, Tartari y Salter, 2011; Colombo y Piva, 2012; Crespi, D'Este, Fontana y Geuna., 2011; Wennberg, Wiklund y Wright, 2011), esto señala la importancia del desarrollo de un perfil emprendedor y no solo técnico.

Conforme a lo anterior, los modelos presentados en las Figuras 3 y 4 que muestran ecosistemas y factores, toman relevancia en la generación de capacidades emprendedoras a los participantes de los equipos de investigación para la transferencia a través de Spin-Off. 
Figura 3

Ecosistema Transformador para Desarrollo de Capacidades Emprendedoras

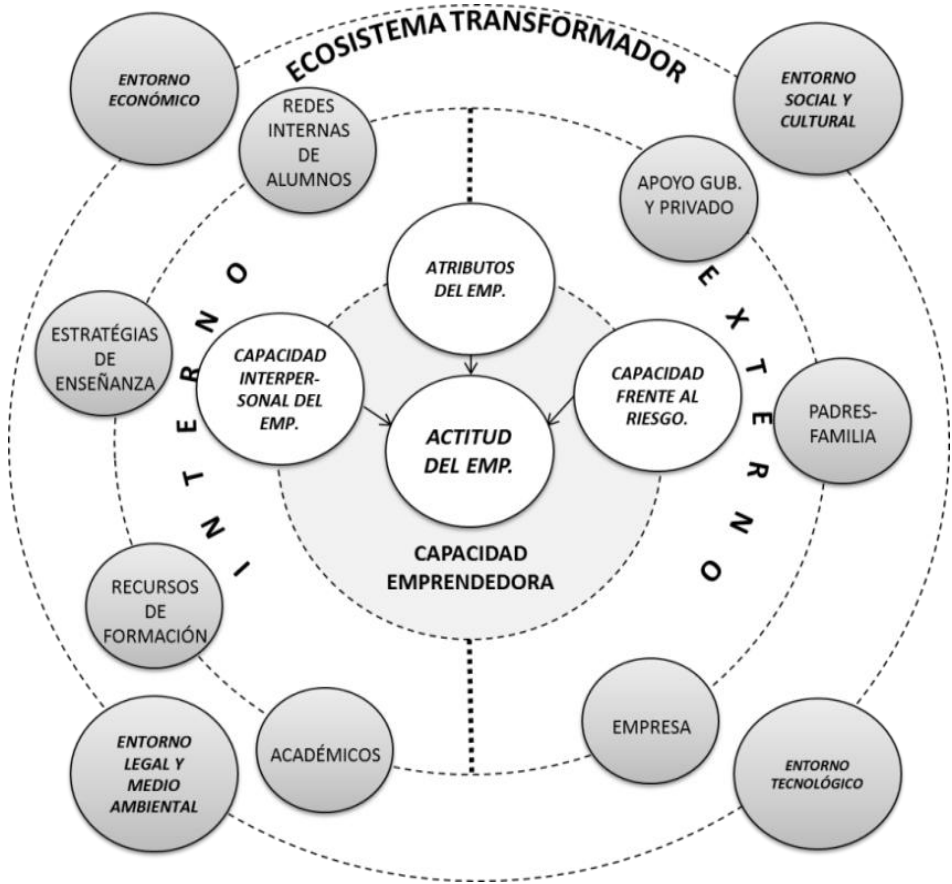

Fuente: Cabana-Villca, Cortes-Castillo, Plaza-Pasten, Castillo-Vergara y Alvarez-Marin (2013)

Figura 4

Factores Determinantes en la generación de Spin-Offs académicas

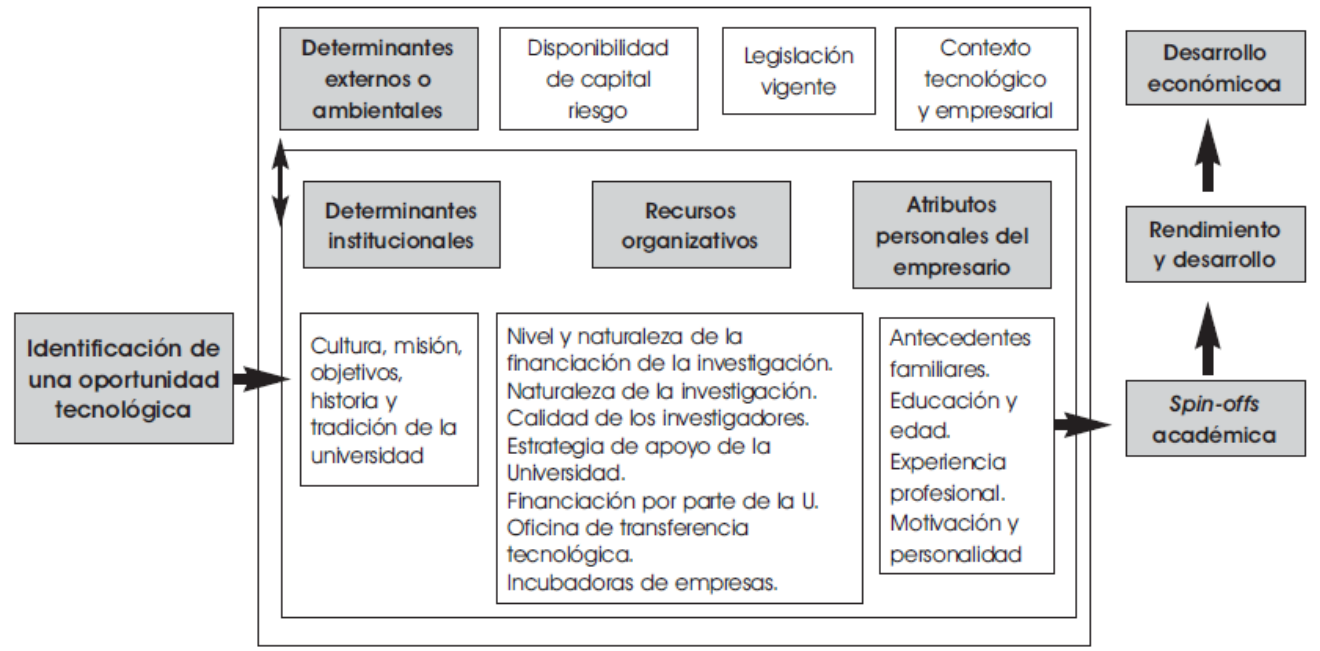

Fuente: Aceytuno y Paz (2008) 
Un ejemplo de Spin-Off es una empresa denominada Energesis Ingeniería, impulsada desde un grupo de investigación denominado Intertech. De los resultados obtenidos del estudio de una instalación geotérmica, se creó en 2004 la empresa Energesis, especializada en el diseño, implantación y gestión de este tipo de instalaciones. Esta empresa es un ejemplo real de cómo la colaboración universidad-empresa es fundamental para que la sociedad se beneficie de los conocimientos e investigaciones realizados en los centros universitarios. Actualmente, el equipo de investigación está formado por más de 30 investigadores adscritos mayoritariamente al Instituto Universitario de Matemática Pura de la Universidad Politécnica de Valencia (UPV), también cuenta con profesionales de la Universitat de València y de la Universidad Católica de Valencia (UCV), con un perfil muy diverso que va desde matemáticos, físicos, ingenieros, biólogos a químicos (Fernández, 2012).

En Chile, también hay casos de Spin-Off, sin embargo, muchas de ellas están asociadas a incubadoras de negocios y no, necesariamente, a los núcleos de investigación de sus académicos. Según Andrade (2014), algunos de estos casos son: Wilefko (Spin-Off de INNOVO de la Universidad de Santiago de Chile, que genera energía eléctrica limpia mediante la extracción de la energía cinética de las olas marinas); Tekemi S.A. (Empresa de ingeniería originada en el Centro de Investigación en Resonancia Magnética de la Pontificia Universidad Católica de Chile); Ideame (Plataforma que ayuda a creadores latinoamericanos a realizar sus proyectos a través de financiamiento colectivo, creada en la incubadora UDD Ventures de la Universidad del Desarrollo); Talent Sistem (Software para el apoyo de selección de personal, incubada en Crece, incubadora de la Universidad Católica del Maule); y Rayen Lemu (Empresa dedicada a la producción y comercialización de hongos comestibles, incubados por Australlncuba de la Universidad Austral).

\section{Conclusiones}

Las universidades en Chile se encuentran inmersas en un proceso de adaptación al nuevo paradigma: el de la universidad empresarial y este está relacionado con la innovación obtenida y al surgimiento y crecimiento de empresas innovadoras. En este proceso, se tornan relevantes los modelos de negocio para comercializar los resultados de investigación que se desarrolla, en las Universidades, primordialmente, con fondos públicos, mediante la formación de Spin-Off. 
En diversos países como Estados Unidos, Reino Unido y España ya se presentan ventajas en torno a estos procesos, se han definido políticas, estructuras y ecosistemas para el desarrollo. Si bien, Chile se destaca como economía con una alta evaluación en los programas de gobierno pro emprendimiento según el Global Entrepreneursgip Monitor del año 2013, carece de una política, estructuras y ecosistemas en educación. Este informe señala que los expertos consideran que la dedicación que emplean las universidades en fomentar el espíritu empresarial y la creación de nuevas empresas no es la adecuada.

Es importante reconocer los esfuerzos gubernamentales pro creación de empresas, pues el gobierno ha impulsado la ley "Empresa en un día" (www.tuempresaenundia.cl) que permite crear una empresa en un día y casi sin costo, así como la ley de quiebras que permite re-emprender después de un fracaso.

El mismo informe señala que en transferencia de investigación, desarrollo y apertura de los mercados, Chile está muy por debajo de las economías basadas en la innovación, aun cuando cada vez más universidades crean centros para el desarrollo de la investigación y transferencia tecnológica. Por lo tanto, es clave que el país desarrolle políticas de apoyo y promoción a la creación de empresas desde las universidades, en conjunto con una legislación que regule los derechos de propiedad intelectual entre las universidades y su personal académico e investigador.

Sin embargo, a la generación de políticas se deben incorporar instancias que fortalezcan el desarrollo, la participación y el papel que juega el equipo investigador, debiendo considerarse estrategias para fomentar las capacidades propias del equipo, más allá del perfil técnico. Entonces, debe considerarse la formación en negocios de estos individuos, o la incorporación de integrantes al equipo que suplan estas competencias, pues se señalan como factor clave de éxito de un Spin-Off.

Hoy las universidades en Chile cuentan, en mayor o menor medida, con estructuras, programas y proyectos dirigidos hacia el fortalecimiento de capacidades emprendedoras en sus estudiantes. Estas estructuras, programas y proyectos se deben poner a disposición de los científicos e investigadores para no duplicar esfuerzos o influir en que se establezcan competencias no deseadas. Las políticas universitarias deben intensificar sus actividades para permitir una cultura emprendedora dentro de las universidades, las políticas en este ámbito deben implicar un cambio importante en la mentalidad universitaria.

En la economía actual, la innovación es un factor esencial para generar desarrollo y mejorar la competitividad de los agentes económicos. Los países que logran generar 
conocimiento y volcarlo hacia innovaciones crecen en mayor medida que aquellos que no, por lo cual resulta imperativo generar ecosistemas tendientes a ello, y uno de los protagonistas principales en este proceso deben ser las Universidades, generando políticas y estrategias tendientes a generar Spin-Off.

\section{Agradecimientos}

Los autores desean agradecer el apoyo de la Dirección de Investigación y del Departamento de Ingeniería Civil Industrial de la Universidad de La Serena.

\section{Referencias}

Aceytuno, Maria y Paz, Manuela. (2008). La creación de Spin-Off Universitarias. El caso de la Universidad de Huelva. Economía industrial, (368), 97-111.

Acosta, Manuel, Coronado, Daniel y Martínez, María. (2012). Spatial differences in the quality of university patenting: Do regions matter? Research Policy, 41(4), 692-703. doi: 10.1016/j.respol.2012.01.002

Agarwal, Rajshree y Shah, Sonali. (2014). Knowledge sources of entrepreneurship: Firm formation by academic, user and employee innovators. Research Policy, 43(7), 11091133. doi: 10.1016/j.respol.2014.04.012

Aldridge, Taylor y Audretsch, David. (2011). The Bayh-Dole Act and scientist entrepreneurship. Research Policy, 40(8), 1058-1067. doi: 10.1016/j.respol.2011.04.006

Andrade, Sebastián. (20 de septiembre, 2014). El auspicioso trabajo de los Spin-Off en chile. Innovación.cl, Recuperado de http://www.innovacion.gob.cl/reportaje/el-auspiciosotrabajo-de-los-Spin-Off-en-chile

Astebro, Thomas, Bazzazian, Navid y Braguinsky, Serguey (2012). Startups by recent university graduates and their faculty: Implications for university entrepreneurship policy. Research Policy, 41(4), 663-677. doi: 10.1016/j.respol.2012.01.004

Bacchiocchi, Emanuele y Montobbio, Fabio. (2009). Knowledge diffusion from university and public research. A comparison between US, Japan and Europe using patent citations. The Journal of Technology Transfer, 34(2), 169-181. doi: 10.1007/s10961-007-9070-y

Beraza, José y Rodríguez, Arturo. (2010). Factores determinantes de la utilización de las Spin-Offs como mecanismo de transferencia de conocimiento en las universidades. Investigaciones Europeas de Dirección y Economía de la Empresa, 16(2), 115-135. doi 10.1016/S1135-2523(12)60115-4

Beraza, José y Rodríguez, Arturo. (2011). Los programas de apoyo a la creación de SpinOffs en las universidades españolas: una comparación internacional. Investigaciones Europeas de Dirección y Economía de la Empresa, 17(2), 89-117. doi: 10.1016/S11352523(12)60054-9 
Beraza, José y Rodríguez, Arturo (2012). Conceptualización de la Spin-Off universitaria, revisión de la literatura. Revista Economía Industrial, (384), 143-152.

Bozeman, Barry, Rimes, Heather y Youtie, Jan. (2014). The evolving state-of-the-art in technology transfer research: Revisiting the contingent effectiveness model. Research Policy, 44(1), 34-49. doi: 10.1016/j.respol.2014.06.008

Bruton, Garry, Ketchen, David e Ireland, Duane. (2013). Entrepreneurship as a solution to poverty. Journal of Business Venturing, 28(6), 683-689. doi: 10.1016/j.jbusvent.2013.05.002

Buenstorf, Guido y Schacht, Alexander. (2013). We need to talk - or do we? Geographic distance and the commercialization of technologies from public research. Research Policy, 42(2), 465-480. doi: 10.1016/j.respol.2012.06.010

Cabana-Villca, Ricardo, Cortes-Castillo, Iván, Plaza-Pasten, Diego, Castillo-Vergara, Mauricio y Alvarez-Marin, Alejandro. (2013). Análisis de las capacidades emprendedoras potenciales y efectivas en alumnos de centros de educación superior. Journal of technology management \& innovation, 8(1), 65-75. doi: 10.4067/S071827242013000100007

Casper, Steven. (2013). The spill-over theory reversed: The impact of regional economies on the commercialization of university science. Research Policy, 42(8), 1313-1324. doi: 10.1016/j.respol.2013.04.005

Clarysse, Bart, Tartari, Valentina y Salter, Ammon (2011). The impact of entrepreneurial capacity, experience and organizational support on academic entrepreneurship. Research Policy, 40(8), 1084-1093. doi: 10.1016/j.respol.2011.05.010

Clarysse, Bart, Wright, Mike, Bruneel, Johan y Mahajan, Aarti. (2014). Creating value in ecosystems: Crossing the chasm between knowledge and business ecosystems. Research Policy, 43(7), 1164-1176. doi: 10.1016/j.respol.2014.04.014

Colombo, Massimo y Piva, Evila. (2012). Firms genetic characteristics and competenceenlarging strategies: A comparison between academic and non-academic high-tech startups. Research Policy, 41(1), 79-92. doi: 10.1016/j.respol.2011.08.010

Conceição, Oscarina, Fontes, Margarida y Calapez Teresa. (2012). The commercialization decisions of research-based Spin-Off: Targeting the market for technologies. Technovation, 32(1), 43-56. doi: 10.1016/j.technovation.2011.07.009

Consejo Nacional de Innovación para la Competitividad. (2010). Evaluation report of national innovation strategy for competitiveness. Santiago, Chile: El Consejo.

Crespi, Gustavo, D’Este, Pablo, Fontana, Roberto y Geuna, Aldo. (2011). The impact of academic patenting on university research and its transfer. Research Policy, 40(1), 5568. doi: 10.1016/j.respol.2010.09.010

Di Gregorio, Dante y Shane, Scott. (2003). Why do Some Universities Generate More Startups than Others? Research Policy, 32(2), 209-227. doi:10.1016/S0048-7333(02)00097-5 
Dias, Alexandre y Porto, Geciane. (2014). Como a USP transfere tecnologia? Organizações \& Sociedade, 21(70), 489-507. doi : 10.1590/S1984-92302014000300008

Dragan, Irina e Isaic-Maniu, Alexandru. (2012). The Entrepreneurship Impact on the Dynamic of Macroeconomic Results. Procedia Economics and Finance, 3, 515-520. doi: 10.1016/S2212-5671(12)00189-X

Fernández, Carlos. (2010). Cómo construir un sistema de transferencia de tecnología en un país en desarrollo. En Gestión de la propiedad intelectual e innovación en Agricultura y en Salud: Un manual de Buenas Prácticas (pp. 199-206). Recuperado de http://pipra.fia.cl/media/9350/f2 6 fern\%C3\%A1ndez vf 13-04-2011.pdf

Fernández, Pedro. (2012). Aplicaciones del modelado matemático en problemas energéticos: un recorrido desde la investigación a la creación de empresas. Revista de la Academia Colombiana de Ciencias Exactas, Físicas y Naturales, 36(138), 93-103.

Fini, Riccardo, Grimaldi, Rosa, Santoni, Simone y Sobrero, Maurizio. (2011). Complements or substitutes? The role of universities and local context in supporting the creation of academic Spin-Offs. Research Policy, 40(8), 1113-1127. doi: 10.1016/j.respol.2011.05.013

García, Teresa y Mulero, Eva. (2007). Medida de los factores claves del éxito de la I+D: el constructo y sus dimensiones. Cuadernos de Economía y Dirección de la Empresa, 10(32), 15-47. doi: 10.1016/S1138-5758(07)70090-7

Genet, Corine, Errabi, Khalid y Gauthier, Caroline (2012). Which model of technology transfer for nanotechnology? A comparison with biotech and microelectronics. Technovation, 32(3-4), 205-215. doi: 10.1016/j.technovation.2011.10.007

Gilsing, Victor, Bekkers, Rudi, Bodas, Isabel y Van der Steen, Marianne. (2011). Differences in technology transfer between science-based and development-based industries: Transfer mechanisms and barriers. Technovation, 31(12), 638-647. doi: 10.1016/j.technovation.2011.06.009

Goldfarb, Brent y Henrekson, Magnus. (2003). Bottom-up versus top-down policies towards the commercialization of university intellectual property. Research Policy, 32(4), 639-658. doi: 10.1016/S0048-7333(02)00034-3

Grimaldi, Rosa, Kenney, Martin, Siegel, Donald y Wright, Mike. (2011). 30 years after BayhDole: Reassessing academic entrepreneurship. Research Policy, 40(8), 1045-1057. doi: 10.1016/j.respol.2011.04.005

Iglesias, Patricia, Jambrino, Carmen y Peñafiel, Antonio. (2012). Caracterización de las SpinOff universitarias como mecanismo de transferencia de tecnología a través de un análisis clúster. Revista Europea de Dirección y Economía de la Empresa, 21(3), 240254. doi: 10.1016/j.redee.2012.05.004

Instituto Nacional de Propiedad Industrial. (2012). Balance de Gestión Integral año 2012. Chile: Ministerio de Economía, Fomento y Turismo. 
Jiménez, Claudia, Maculan, Ane, Otálora, Ivone, Moreira, Robson y Castellanos, Oscar. (2013). Reflexiones sobre los mecanismos de transferencia de conocimiento desde la universidad: el caso de las Spin-offs. Recuperado de http://www.altec2013.org/programme pdf/1119.pdf

Kenney, Martin y Patton, Donald. (2011). Does inventor ownership encourage university research-derived entrepreneurship? A six university comparison. Research Policy, 40(8), 1100-1112. doi: 10.1016/j.respol.2011.05.012

Lehoux, Pascale, Daudelin, Genevieve, Williams-Jones, Bryn, Denis, Jean-Louis y Longo, Christopher. (2014). How do business model and health technology design influence each other? Insights from a longitudinal case study of three academic Spin-Offs. Research Policy, 43(6), 1025-1038. doi: 10.1016/j.respol.2014.02.001

Leten, Bart, Landoni, Paolo y Van Looy, Bart. (2014). Science or graduates: How do firms benefit from the proximity of universities? Research Policy, 43(8), 1398-1412. doi: 10.1016/j.respol.2014.03.005

Meneses, Francisco. (2009). Difusión y Transferencia Tecnológica. Chile: Innova Chile.

Ndonzuau, Frederic, Pirnay, Fabrice y Surlemont, Bernard. (2002). A stage model of academic Spin-Off creation. Technovation, 22(5), 281-289. doi: 10.1016/S01664972(01)00019-0

O'kane, Conor, Mangematin, Vincent, Geoghegan, Will y Fitzgerald, Ciara. (2014). University technology transfer offices: The search for identity to build legitimacy. Research Policy, 44(2), 421-437. doi: 10.1016/j.respol.2014.08.003

Ortín-Ángel, Pedro y Vendrell-Herrero, Ferran. (2014). University Spin-Offs vs. other NTBFs: Total factor productivity differences at outset and evolution. Technovation, 34(2), 101112. doi: 10.1016/j.technovation.2013.09.006

Rasmussen, Einar y Wright, Mike. (2015). How can universities facilitate academic Spin-Offs? An entrepreneurial competency perspective. The Journal of Technology Transfer, 1-18. doi: 10.1007/s10961-014-9386-3

Rasmussen, Einar, Mosey, Simon y Wright, Mike. (2014). The influence of university departments on the evolution of entrepreneurial competencies in Spin-Off ventures. Research Policy, 43(1), 92-106. doi: 10.1016/j.respol.2013.06.007

Schwab, Klaus. (2014). The Global Competitiveness Report 2014-2015. World Economic Forum. USA: Columbia University.

Soetanto, Danny y Van Geenhuizen, Marina. (2015). Getting the right balance: University networks' influence on Spin-Offs' attraction of funding for innovation. Technovation, 3637(0), 26-38. doi: 10.1016/j.technovation.2014.10.008

Steffensen, Morten, Rogers, Everett y Speakman, Kristen. (2000). Spin-Offs from research centers at a research university. Journal of Business Venturing, 15(1), 93-111. doi: 10.1016/S0883-9026(98)00006-8 
Sternberg, Rolf. (2014). Success factors of university-Spin-Offs: Regional government support programs versus regional environment. Technovation, 34(3), 137-148. doi: 10.1016/j.technovation.2013.11.003

Tongur, Stefan y Engwall, Mats. (2014). The business model dilemma of technology shifts. Technovation, 34(9), 525-535. doi: 10.1016/j.technovation.2014.02.006

Treibich, Tania, Konrad, Kornelia y Truffer, Bernhard. (2013). A dynamic view on interactions between academic Spin-Offs and their parent organizations. Technovation, 33(12), 450462. doi: 10.1016/j.technovation.2013.06.012

Van Looy, Bart, Landoni, Paolo, Callaert, Julie, Van Pottelsberghe, Bruno, Sapsalis, Eleftherios y Debackere, Koenraad. (2011). Entrepreneurial effectiveness of European universities: An empirical assessment of antecedents and trade-offs. Research Policy, 40(4), 553-564. doi: 10.1016/j.respol.2011.02.001

Vedovello, Conceição y Figueiredo, Paulo (2005). Incubadora de inovação: que nova espécie é essa? RAE eletrônica, 4(1). doi: 10.1590/S1676-56482005000100006

Visintin, Francesca y Pittino, Daniel. (2014). Founding team composition and early performance of university-Based Spin-Off companies. Technovation, 34(1), 31-43. doi: 10.1016/j.technovation.2013.09.004

Walter, Achim, Auer, Michael y Ritter, Thomas. (2006). The impact of network capabilities and entrepreneurial orientation on university Spin-Off performance. Journal of Business Venturing, 21(4), 541-567. doi: 10.1016/j.jbusvent.2005.02.005

Wennberg, Karl, Wiklund, Johan y Wright, Mike. (2011). The effectiveness of university knowledge spillovers: Performance differences between university spinoffs and corporate spinoffs. Research Policy, 40(8), 1128-1143. doi: 10.1016/j.respol.2011.05.014 